\title{
The Visual Brain: Computing Through Multiscale Complexity
}

\author{
Yves Frégnac, Julien Fournier, Florian Gérard-Mercier, Cyril Monier, \\ Marc Pananceau, Pedro Carelli, and Xoana Troncoso
}

\begin{abstract}
Information coding in sensory neurons is both digital, in terms of neuronal output spike timing and rate, and analog, produced by the irregular subthreshold changes in somatic and dendritic membrane potential resulting from synchronized volleys of synaptic inputs. Intracellular recordings give a unique access to a composite multiscale signal where the local microscopic integration process realized by a single neuron can be studied in the global mesoscopic context of the "unseen" units afferent to the recorded cell. This chapter shows how reverse engineering approaches can be used in the primary visual cortex of higher mammals to reveal the hidden complexity of visual processing and establish causal links between the functional dynamics of synaptic echoes in primary visual cortex and perceptual biases in low-level, non-attentive perception.
\end{abstract}

\section{Introduction}

A still highly controversial issue in visual perception concerns the definition of receptive fields (RFs). Do they represent a static transfer function associated with each sensory neuron or a dynamic integration window open on the sensory world and continuously reshaped by contextual changes in the input statistics? How do RF properties adapt to contrast/luminance non-stationarities experienced during the oculomotor exploration of the visual world? Our understanding of early visual processing (implemented in our brain by the retino-thalamo-cortical projection)

\footnotetext{
Y. Frégnac $(\bowtie) \cdot$ F. Gérard-Mercier • C. Monier • M. Pananceau • P. Carelli • X. Troncoso Centre National de la Recherche Scientifique (CNRS-UNIC), Unité de Neuroscience, Information et Complexité (UNIC), Gif-sur-Yvette, France

e-mail: fregnac@unic.cnrs-gif.fr

\section{J. Fournier}

Centre National de la Recherche Scientifique (CNRS-UNIC), Unité de Neuroscience, Information et Complexité (UNIC), Gif-sur-Yvette, France
}

Max Planck Institute for Brain Research, Max von Laue Str. 4, 60438 Frankfurt am Main, Germany 
in higher mammals has been shaped, to a large degree, with a hypothesis-driven conception of sensory architecture in mind. This bias is best illustrated by the 50 -year dominance of the hierarchical schema of genesis of RF specificity (Hubel and Wiesel 1962, 1968, 2005), where visual attribute extraction in the early visual system results from a serial processing starting from non-oriented retinal and thalamic RFs to a cascade of oriented edge detectors of increasing complexity across multiple successive relays (thalamus, primary and secondary cortical areas). This feedforward view of visual processing is based on the repetition, at each stage of integration, of canonical, but highly specific, rules of anatomical convergence from which the function derives. Consequently, our current understanding of RF genesis is largely bottom-up driven and contradicts Aristotle's principle that "the whole is greater than the sum of its parts." Although this simplifying view of sensory processing has led to major advances [review in Alonso (2002); see also the remarkable obituary tribute to David Hubel by Kevan Martin (2014)], it fails to account for the functional complexity expected from the recurrent structural connectivity of cortical subcircuits (Douglas and Martin 2004) on the one hand and the non-linear nature of the dynamic interactions between excitation and inhibition during sensory processing (Borg-Graham et al. 1998; Monier et al. 2008). Furthermore, as emphasized by Bruno Olshausen (2014), RF classification has been established using highly standardized and parametrized sensory contexts (spots, bars and gratings), which have little to do with the rich spatio-temporal statistics experienced during the natural scene-viewing conditions of our everyday life. The principle of maximization of a single neuron's firing rate, which initially guided the "neuronal doctrine" (Barlow 1972), does not hold any more when RFs are engaged by non-optimal stimuli most of the time, leading, for natural scene processing, to a sparser and unexpectedly temporally precise spiking regime in the primary visual cortical area (Vinje and Gallant 2000; Baudot et al. 2013). A third conceptual limit to our present knowledge of early visual processing is that most modeling efforts have been targeted at explaining sensory discharges only at the spike level in a purely phenomenological perspective (see Carandini et al. 2005 for a review) rather than aiming at elucidating causal, conductance-based mechanisms regulating the temporal selectivity of the spiking opportunity window (Haider et al. 2010; Baudot et al. 2013).

Although in vivo intracellular recordings are difficult to practice in higher mammals, and thus often limited to the anesthetized and paralyzed preparation (but see Tan et al. 2014 for a "tour de force" in the behaving non human primate), they still appear to be the technique of choice to address quantitatively the synaptic nature of the RF (Fig. 1a, b). In contrast to most imaging methods (voltage-sensitive dye, two-photon) or correlation studies based on multiple recordings, intracellular recordings allow us to differentiate the local microscopic integration process achieved by the single neuron from the more mesoscopic contribution of the network of unseen units that influence at each point in time the activity of the recorded cell (Fig. 1c).

We review here recent intracellular electrophysiological studies from the Frégnac lab (CNRS-UNIC), done in the visual cortex of the anesthetized 

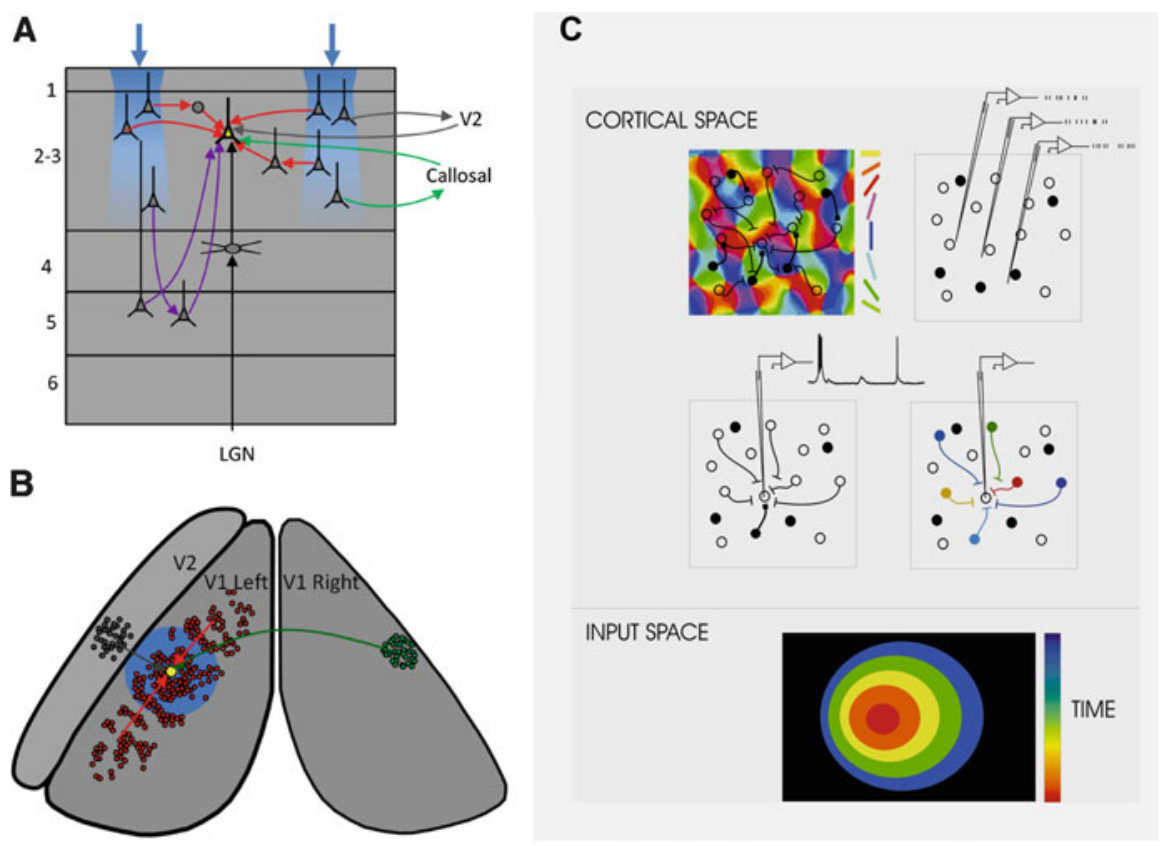

Fig. 1 Bridging microscopic and mesoscopic scales. (a) The synaptic RF (adapted from Huang et al. 2014). Sensory cortical neurons integrate the feedforward drive from the thalamus (LGN), eventually relayed by intracolumnar connections and amplified by recurrent local connectivity, with the lateral input provided by intrinsic, horizontal long-distance connections and corticocortical feedback and interhemispheric callosal loops. (b) Retrieving mesoscopic dynamics from intracellular recordings (adapted from Frégnac et al. 2007). Top row, classical methods for studying evoked sensory dynamics: left, voltage-sensitive, dye-imaging map based on hemodynamic signals; right, same network analyzed with simultaneous multiple electrode recordings (blind connectivity). Middle row, intracellular recording where reverse engineering methods allow extraction of the "effective connectivity," influencing the membrane potential at any point in time. Synaptic functional imaging, based on feature selectivity and space and time, allows us to identify the synaptic sources and reconstruct predictions of the full network dynamics (bottom row)

mammalian brain and addressing separately two scales of spatial integration in the V1 RF: (1) the inner ON-OFF organization of the RF core (underlying the "Simple" vs. "Complex" typology), depending on the balance between feedforward and local recurrent connectivity, and (2) the "association field" extending in the "silent" surround of the RF, from which subthreshold activation can be evoked through the propagation along long-distance, slowly conducting "horizontal" connections intrinsic to V1 (Bringuier et al. 1999; Chavane et al. 2011; Frégnac 2012; GérardMercier et al. in preparation).

The claim we make here is that the intracellular subthreshold membrane potential signal gives unique access to the multiscale nature of cortical processing and that reverse engineering methods can be designed to unfold, from the intracellular reading of synaptic echoes, the mesoscopic dynamics of the afferent network 
(Fig. 1, right panel). In the first part of this review, we will show that the hidden complexity revealed by this approach demonstrates how limited our current understanding is of the bottom-up emergence of dynamic properties in visual RFs in the early visual system. Furthermore, it unravels the existence of immergence processes through which the collective mesoscopic constraints imposed by the distributed sensory input regulate the functional expression of individual RF properties in a top-down fashion. In the second part, we will illustrate how the decoding of synaptic echoes originating from the silent surround of the RF allows us, in a surprising way, to extract functional structural biases that may serve the selforganization of psychological Gestalt laws in the non-attentive brain. These last findings can be seen as one of the few successful attempts to link visually evoked synaptic dynamics to perceptual biases and low-level perception, thus establishing a causal bridge between microscopic and macroscopic scales.

\section{The Synaptic Imprint of Mesoscopic "Immergence" in Visual RFs}

RFs in primary visual cortex (V1) are categorized as "Simple" or "Complex," depending on their spatial selectivity to stimulus contrast polarity, leading to the retinotopic segregation (Simple) or overlap (Complex) of "ON" and "OFF" subfields (Hubel and Wiesel 1962, 1968). Since the pioneering work of Hubel and Wiesel in the cat and macaque visual cortex, most extracellular surveys have reported V1 RFs with intermediate behaviors (Henry 1977). Intracellular recordings have shown that the separation between Simple and Complex RFs hides a continuous distribution of synaptic inputs, in particular with regard to their degree of linearity (in the sense of system theory; Skottun et al. 1991; Priebe et al. 2004). In spite of the general acceptance that Simple and Complex RF organizations correspond to distinct balance levels between linear and nonlinear contributions at the synaptic level, few studies have investigated whether the functional expression of the Simple or Complex nature of V1 RFs depends, in the same cell, on the spatiotemporal statistics of the stimulus. This may be surprising, since gain-control mechanisms are known to ensure the contrast invariance of the cell selectivity, and it is well established that adaptation to stimulus contrast does not have the same effect on the Simple-like and Complex-like components of V1 cell responses when assessed with drifting gratings. Still, most previous studies of RF adaptation to visual statistics focused either on linear RF components in Simple cells or on nonlinear components in Complex cells and not on the differential adaptation of these two components in the same RF (but see Yeh et al. 2009).

In a series of studies targeting the subthreshold definition of the RF, we realized that RF maps varied significantly between stimulus conditions, leading us to reassess in a quantitative way how the Simple/Complex nature of individual V1 cells depends on the full field visual context used to probe the RF (Fournier 
et al. 2011, 2014). This was done by comparing systematically, in the same cell recorded intracellularly, the synaptic responses to three classical RF mapping protocols based on white noise: sparse noise, ternary dense noise and flashed Gabor noise. A surprising result was that the linear kernel estimate differed between these various contextual noises, in contrast with the prediction of invariance made by cascade L-N-P models of V1 RFs (according to the so-called Bussgang theorem; Bai et al. 2007). Intracellular recordings revealed that, for most V1 cells, there was no such thing as an invariant RF type, but that the relative weights of Simple-like and Complex-like RF components were scaled such as to make the same RF more Simple-like with dense noise stimulation and more Complex-like with sparse or Gabor noise stimulations (example in Fig. 2a; population analysis in Fig. 2b). However, once these context-dependent RFs were convolved with the corresponding stimulus, the balance between Simple-like and Complex-like contributions - in terms of input current- to the synaptic responses appeared to be invariant across input statistics (Fig. 2c; Fournier et al. 2011).

This invariance of the ratio between the linear/nonlinear input current contributions suggests a novel form of homeostatic control of V1 functional properties, where the expressed network nonlinearities are optimized by the statistical structure of the visual input. This study is the first, to the best of our knowledge, to show such clear changes in terms of spatiotemporal reorganizations of synaptic and discharge fields at the single cell level, interpretable as a coherent adaptive behavior at the cortical population level. The claim made here is that these effects are more detectable at the subthreshold than at the spiking level, where additional static non-linearities may interfere with the global read-out of the connectivity adaptation rule.

A functional interpretation of these data could be that the Simple or Complex nature of V1 RFs arises from a variable balance between feed-forward and lateral inputs, with the feed-forward drive providing the Simple-like component whereas the recurrent lateral connections would convey Complex-like contributions (Fig. 3, left). Accordingly, the results might be explained by the functional recruitment of lateral interactions in sparse stimulation conditions and by the decoupling of adjacent cortical columns in dense visual contexts. This view is supported by other studies, realized for instance by the group of Matteo Carandini, suggesting that the lateral propagation of activity between adjacent cortical units decreases substantially when the stimulus contrast is increased (Fig. 3, right, adapted from Nauhaus et al. 2009). In view of these different results, the stimulus dependence of the lateral cortical interactions likely generalizes to other stimulus dimensions, rather than remaining exclusive to the local contrast. Similar effects might be obtained by increasing the spatial or temporal density of the stimulus, with the important parameter probably being the effective contrast along the stimulus feature dimensions for which the cell is selective.

To enrich the predictive power of the synaptic RF model, we decomposed the second-order kernel estimate obtained by a truncated Volterra expansion of the membrane potential response to dense noise, into a non-linear combination of parallel Simple-like filters in a way similar to the spike-triggered covariance (STC) introduced by the groups of Simoncelli and Movshon (Rust et al. 2005). 


\section{A ON/OFF decomposition}

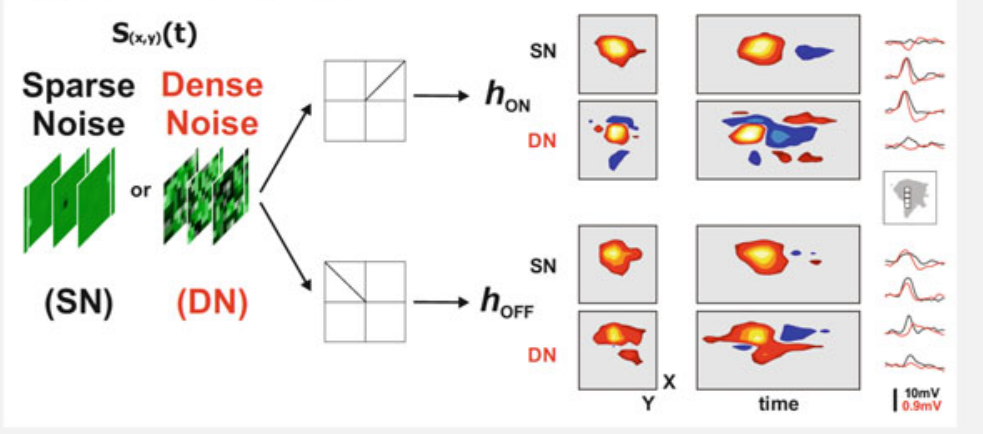

B

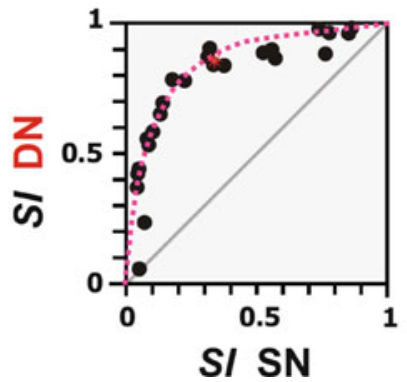

C

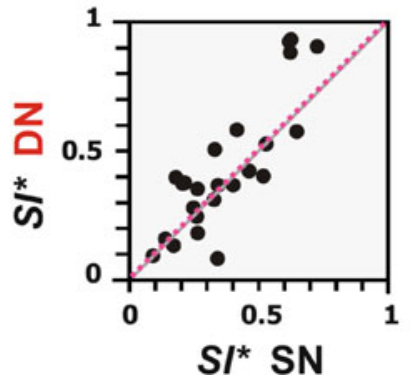

Fig. 2 The functional expression of V1 RFs depends on input statistics. (a) Example of a layer 2-3 cell: the ON and OFF kernels are shown for the two noise input statistics used to map the subthreshold RF ( $S N$ sparse noise, $D N$ dense noise). The shaded boxes represent the $\mathrm{X}-\mathrm{Y}$ and $\mathrm{X}$-time features of the RF filter, with $\mathrm{ON}$ and OFF subfields represented in red and blue, respectively. Note that the maps are Simple-like for the SN statistics [spatially segregated ON and OFF subfields (X-Y map) and reversal of the spatio-temporal filter polarity with time (X-t profile)] and Complex-like for the DN statistics (spatially overlapping subfields). Right column, the individual kernel waveforms $(\mathrm{mV})$, detailed for four different pixels (inset), are represented in red for DN and black for SN. Note the divisive effect of dense noise compared to sparse noise on the kernel estimate amplitude (by a tenfold factor). (b, c) Population analysis of the stimulus dependency of the Simpleness Index (given by the ratio of the linear kernel energy divided by the total RF kernel energy). " 0 " stands for Complex RFs (purely non-linear) and "1" for Simple RFs (purely linear). (b) Population bi-histogram plot linking (on a cell-by-cell basis) the Simpleness Index (SI) for sparse noise (SN, abscissa) and dense noise (DN, ordinate) stimulation. Hyperbolic fit as a pink dotted curve. (c) Same population bi-histogram for $\mathrm{SI}^{*}$ values obtained after convolution of the kernels with the visual input waveform. Note the realignment of the points (cells) along the identity line (bihistogram diagonal). See details in Fournier et al. (2011)

Although the STC method was applied with success at the spiking level to reveal non-linear subunits in V1 and MT cells in the macaque, it failed to reveal more diversity in the cat (Touryan et al. 2002, 2005), probably for technical reasons linked to the limited number of spikes. This potential problem is bypassed here by applying similar techniques to the continuous intracellular membrane potential 


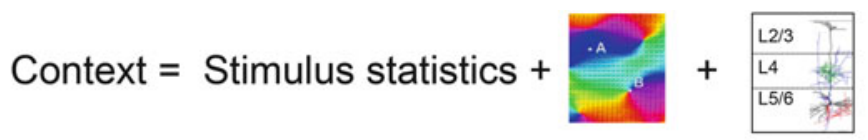
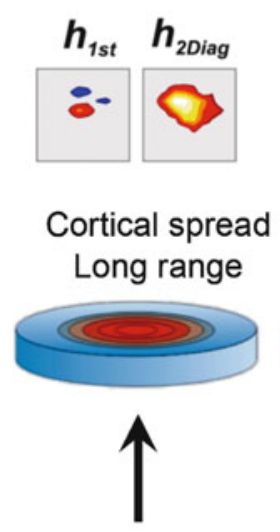

Sparse input

Fournier, Monier, Pananceau and Frégnac, 2011

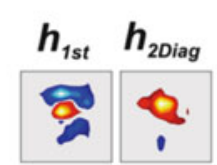

Cortical spread

Short range
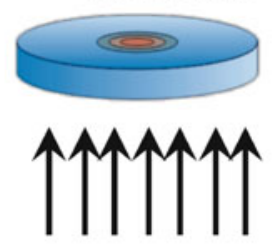

Dense input

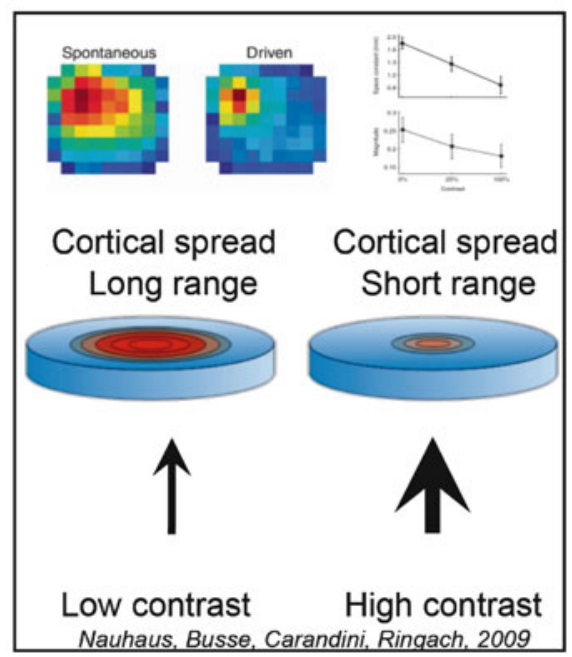

Fig. 3 Immergence of mesoscopic network organization on microscopic response properties. Changes in the mesoscopic context are provided by, functionally, non-stationarities in the sensory input drive statistics and, structurally, by non-homogeneities in the columnar and lateral network neighborhood (Monier et al. 2003). (A) Homeostasis Rule as a function of spatial and temporal density (Fournier et al. 2011): regulation of the functional expression of the RF (h1st for linear and h2nd Diag for the diagonal term of the second-order kernel) by input statistics (left, SN: right, DN). The spread of lateral activation is wider for SN than for DN. (B) Homeostasis Rule as a function of contrast level (adapted from Nauhaus et al. 2009): similar regulation of the lateral spread through horizontal connectivity by the stimulus contrast

signal, resulting in a much higher sensitivity for detecting stimulus-evoked subthreshold events contributing to the spike discharge (Fournier et al. 2014). Conductance or current-based principal component analysis (PCA) allows us to decompose the Simple-like and Complex-like components of the subthreshold RF into a parallel set of functionally distinct subunits, consisting of a Simple-like sub-unit whose linear output accounts for the push-pull components of the RF and a variable number of Complex-like sub-units that contribute in a fully rectified manner to the cell response (Fig. 4, left). Results show that both Simple and Complex RFs exhibit a remarkable diversity of excitatory and inhibitory Complex-like contributions, which differ in orientation and spatial frequency selectivity from the linear RF, even in layer 4 and layer 6 Simple cells that received direct input from the thalamus (exemplified in Fig. 4, right). Furthermore, the diversity of Complex-like contributions recovered at the subthreshold level is expressed in the cell spiking output.

These results demonstrate that the Simple or Complex nature of V1 RFs does not rely on the diversity of Complex-like components received by the cell from its 


\section{Second-order decomposition of V1 synaptic RFs}
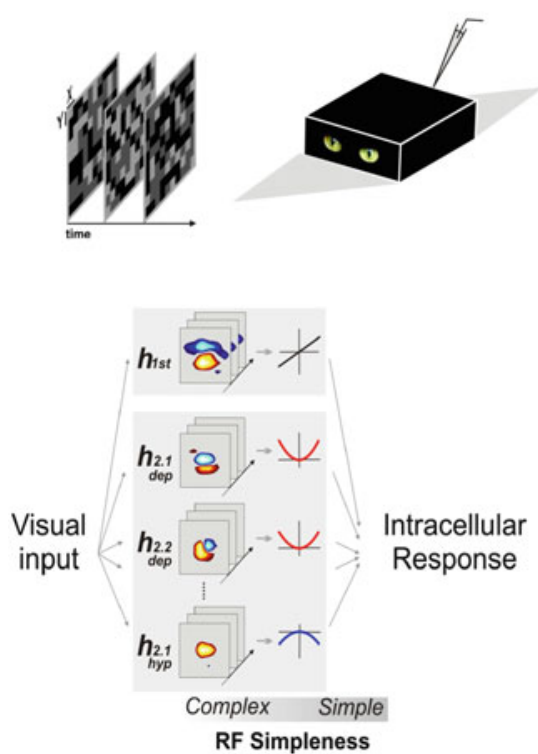
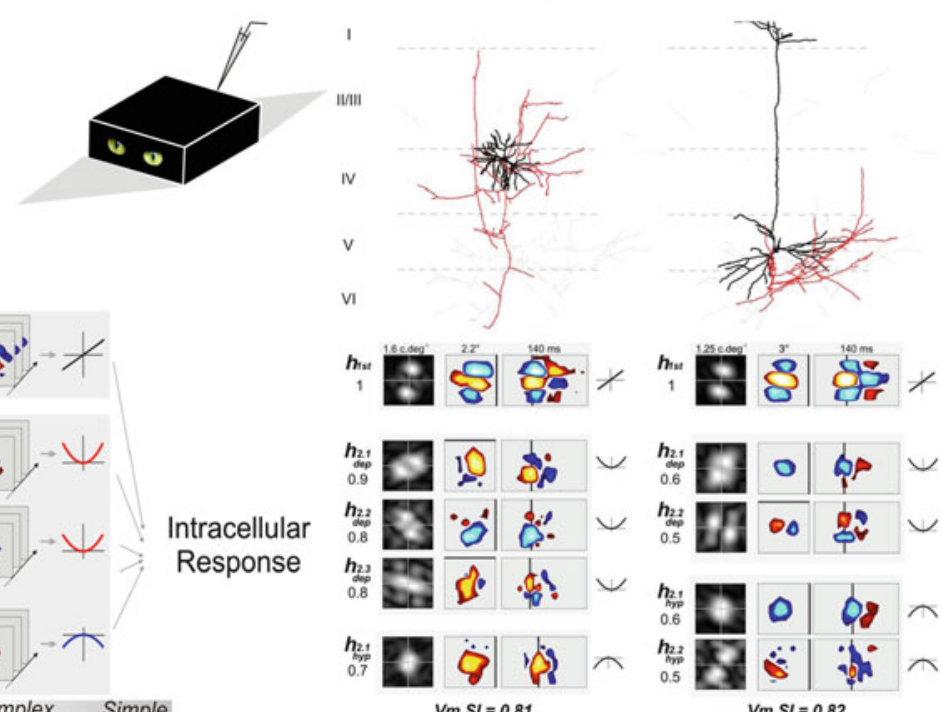

$V m S I=0.81$

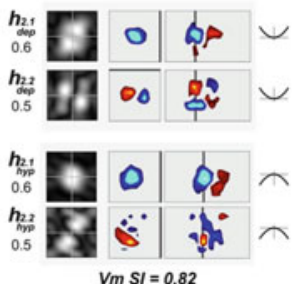

Fig. 4 Filter bank decomposition of the subthreshold V1 RF (adapted from Fournier et al. 2014). Left: decomposition principle: each branch of the filter bank is composed of a Simple-like filter followed by an identity contrast function (linear kernel, upper branch) and by a parallel bank of linear subunits feeding excitatory (red) and inhibitory (blue) quadratic contrast-dependent non-linearities (lower parallel branches). Right: example of RF decomposition for two biocytin reconstructed cells in, respectively, layer 4 (middle) and at the border between layers 5/6 (right) in cat visual subunit. Each subunit weight (in the decomposition) is given below each kernel component

synaptic afferents but on the relative imbalance between the weights of the Simplelike and Complex-like synaptic contributions. In spite of the likelihood that the Simple-like RF subunit results from the push-pull arrangement of excitatory and inhibitory feedforward inputs selective for the same orientation, the diversity of feature selectivity expressed by the Complex-like RF subunits is not consistent with a strict iso-orientation preference rule for excitatory and inhibitory input conductance as generally posited (Ferster and Miller 2000; Priebe and Ferster 2012). Although the estimated Complex-like subunits are operational filters that do not necessarily correspond to the RFs of neurons presynaptic to the recorded cell, they bear a striking resemblance to the linear RF of V1 Simple cells, which suggests that they could correspond to separate subcircuits originating from within the cortex (Rust et al. 2005; Chen et al. 2007). The diversity of orientation and spatial frequency preferences of the Complex subunits agrees with that found in the tuning of the excitatory and inhibitory input conductances measured by voltage clamp techniques in vivo and previously reported by our lab (Monier et al. 2003, 2008). Taken together, these intracellular results support the hypothesis that the Complexlike components of V1 RFs arise from lateral interactions between adjacent cortical 
columns and are consistent with the proposal that the Simple or Complex nature of V1 RFs arises from the respective balance between feedforward and lateral connectivity (Chance et al. 1999; Tao et al. 2004). This wide functional spectrum of Complex-like synaptic contributions to both Simple and Complex RFs may constitute the skeleton of a multi-competent substrate allowing V1 cells to adapt on-thefly to the abrupt changes in the spatio-temporal statistics of visual inputs (Fig. 4, right).

\section{Synaptic Correlates of Perceptual Gestalt Laws in the "Silent Surround" of V1 RFs}

The synaptic RF stems from the interplay of distinct sets of connections, the feedforward drive from the thalamus relayed eventually by vertical processes within the cortical column, the local recurrent reverberation usually confined within a hypercolumn, the long-distance connectivity intrinsic to V1 (that may even originate from the other hemisphere through the corpus callosum) and the feedback from higher cortical areas (Fig. 1a, b). The cat and ferret visual cortex appear to be ideal experimental models to study horizontal connectivity (Kisvardày et al. 1997; Bosking et al. 1997), since many reconstructed axons of pyramidal cells remaining within the gray matter have been shown to extend over several hypercolumns (up to 6-8 mm in the cat; Kisvardày et al. 1997; Callaway and Katz 1990; Gilbert and Wiesel 1983; Gilbert and Li 2012; Buzàs et al. 2006; but see Martin 2014). In spite of some pioneering attempts (Kasamatsu et al. 2010; Mizobe et al. 2001), only limited physiological data have addressed the synaptic contribution of the "silent" surround of the classical V1 RFs, from which impulse-like stimuli fail to evoke a spiking response. Consequently, the role of long distance horizontal connectivity in influencing the response gain within the classical RF, and in particular in boosting it for specific center-surround stimulus conditions (Jones et al. 1980; Sillito et al. 1995; Sillito and Jones 1996), remains an issue of debate. In spite of this uncertain status, horizontal connectivity has long been presented as the biological substrate of iso-preference binding in the electrophysiological and psychophysical cortical literature (review in Gilbert and Li 2012; Frégnac and Bathellier 2015). This principle was derived from a developmental rule that posited that "who fires together (or is alike) tend to wire together" (Callaway and Katz 1990). At the psychophysical level, this view corresponds to the perceptual "association field" concept, developed by Field, Hess and their colleagues in the 1990s (Field et al. 1993). This concept assumes the instantaneous induction of collinear and, to a lesser extent, co-circular facilitation by the static presentation of oriented contrast edges. This elegant psychophysical hypothesis accounts in humans for the "popout" perception of smooth contiguous path integration even when immersed in a sea of randomly oriented edge elements (Fig. 5a, top; Field et al. 1993) and the facilitation of target detection by high contrast co-aligned flankers (Fig. 5a, bottom; 
A
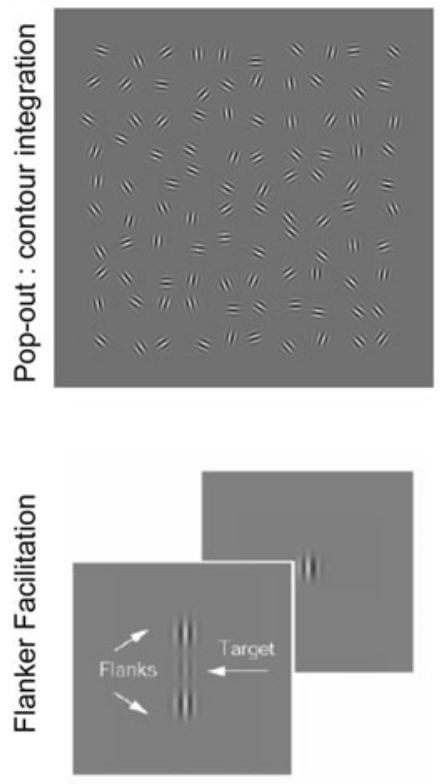

B

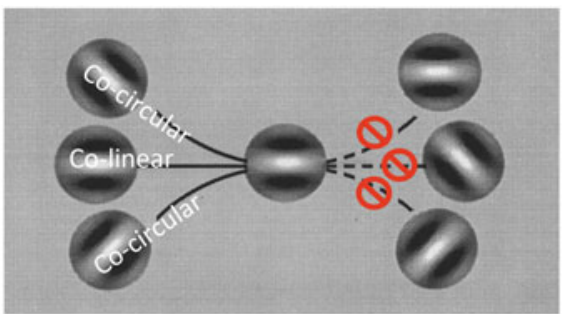

C

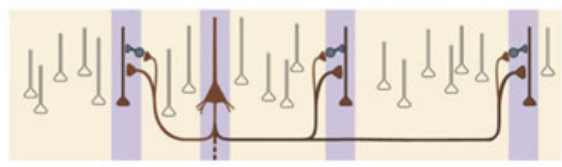

Ansol
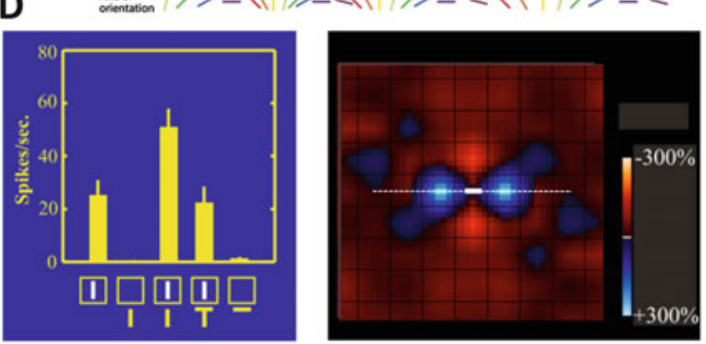

Fig. 5 The perceptual association field and its neuronal correlate in the attentive brain (reviewed in Frégnac and Bathellier 2015). (a) Top: "pop-out" emergence of a continuous integration path in a sea of randomly oriented Gabor patches (Field et al. 1993). Bottom: facilitation of detection of a low contrast vertical Gabor element induced by the simultaneous presentation of co-aligned high contrast flanker elements (Polat and Sagi 1993). (b) Hypothetical association field induced by an oriented element through lateral interactions promoting co-alignment and co-circularity (Field et al. 1993). (c) The "iso-functional binding" hypothesis (Gilbert and Li 2012). An individual superficial layer cortical pyramidal cell forms long-range connections that extend many millimeters parallel to the cortical surface. Long-range connections ( $>500 \mu \mathrm{m}$ from the injection center) tend to link columns of similar orientation preference. (d) The "neural facilitation field" (Li et al. 2006). Left, the responses of V1 neurons are amplified in the awake behaving monkey by collinear contours extending outside the RF. Introducing a cross-oriented bar between the collinear segments blocks the contour-related facilitation. Right, two-dimensional map of facilitatory (blue) and inhibitory (red) modulation of the response to an optimally oriented line segment centered in the RF (horizontal white bar). The spiking modulation is suppressed by anesthesia

Polat and Sagi 1985). At the neuronal level, this view is supported by the peculiar anatomy of long-distance horizontal connections emitted by supragranular pyramidal cells found consistently in higher mammals (but see Martin et al. 2014) and the electrophysiological demonstration of a "neural facilitation field" (Fig. 5b, c; Gilbert and Li 2012). These latter experiments, realized in the attentive behaving monkey, demonstrated an impressive boosting of the response gain to an optimally oriented contrast edge within the classical RF when flankers were simultaneously flashed in the "silent surround" and co-aligned along the preferred orientation axis of the extracellularly recorded cell. Most remarkably, Charles Gilbert, Wu Li and his colleagues showed that, to be expressed, the co-linearity binding rule required 
the existence of top-down signals, present in the target-attending monkey, since the effect was weakened by diverted attention ( $\mathrm{Li}$ et al. 2006) and the ability to learn contour integration was suppressed by anesthesia (Li et al. 2008).

These previous studies provided, nevertheless, an indirect answer since they addressed only the modulatory nature of the center-surround effects, without probing the existence of a subthreshold influence. This issue has been addressed intracellularly in the anesthetized mammal, and our lab has demonstrated repeatedly, in the context of various stimulation protocols, the existence of long-distance propagation of visually evoked activity through lateral (and possibly feedback) connectivity outside the classical RF (Bringuier et al. 1999; Frégnac 2012; GerardMercier et al. 2016; Troncoso et al. 2015). This propagation, initially hypothetized by Amiram Grinvald and inferred from the synaptic echoes we recorded intracellularly, has since been confirmed in the same species by voltage sensitive dye (VSD) imaging techniques (Benucci et al. 2007; Chavane et al. 2011), which provide a direct visualization of the horizontal propagation pattern at the mesoscopic level of the V1 retinotopic map. Most remarkably, the VSD waves were found to travel at the same speed as that inferred from intracellular recordings $(0.3 \mathrm{~m} / \mathrm{s})$.

In a recent intracellular study (Gérard-Mercier et al. 2014, in preparation), we reinvestigated the association field concept to demonstrate whether a structurefunctional bias might be still detected at the subthreshold level, even in the absence of attention-related signals. By averaging synaptic response properties in a unified "cellulo-centric" reference frame centered on the discharge field center and realigned with the spike-based orientation preference, we found a coherent spatial organization of visual synaptic responses, reflecting the grouping bias of the "perceptual association field" for collinear contours (Field et al. 1993). This result, apparently contradictory to Gilbert and Li's failure to find the "facilitatory neural field" under anesthesia, is seen only at the population level by summation across cells. The most likely interpretation is that a mean-field effect (in the sense of physics) is needed to enhance a slight bias in the subthreshold impact of the synaptic connectivity intrinsic to V1. Its expression is revealed (or facilitated) here by the use of $3-4^{\circ}$ test-oriented stimuli (Gabor patch) that recruit by spatial summation the whole extent of the aggregate RF of a hypercolumn in the cat. Our current working hypothesis is that a critical threshold of spatial synergy and temporal summation has to be trespassed to make the weak functional impact of these long-range interactions (in the $\mathrm{mV}$ range) detectable, as suggested from a prior combined VSD and intracellular study done in collaboration with the lab of Amiram Grinvald (Chavane et al. 2011). Preliminary intracellular data show that two- to six-stroke apparent motion (AM) sequences, riding in phase with horizontal activation in a centripetal way towards the RF center, are effective enough to unmask suprathreshold filling-in responses in the unstimulated RF core (Troncoso et al. 2015).

Our work provides, for the first time, intracellular evidence in the anesthetized mammal for synaptic correlates of low-level perception, closely dependent on the spatiotemporal features of the synaptic integration field of V1 neurons and most 
likely linked to intra-V1 horizontal connectivity. These findings also agree with the concept of a "dynamic association field," whose spatial anisotropy and extent are transiently updated and reconfigured as a function of changes in the retinal flow statistics imposed during visuomotor exploration of natural scenes (Frégnac 2012). According to this still hypothetical view, the propagation of intracortical depolarizing waves at the mesoscopic V1 map level would help in broadcasting an elementary form of collective predictive "belief" to distant parts of the network, at a time when they are not yet engaged by the stimulus drive. We propose that the in-phase association of horizontal and feedforward input could provide the synaptic substrate for implementing the psychological Gestalt principles of common fate and axial collinearity (review in Wagemans et al. 2012). On a more conjectural note, since a visual flow in the order of $100-250 \%$ in retinal space is needed to maintain - in cat V1 - the feedforward flow in phase or slightly ahead of intra-V1 propagation, one may expect the amplification of visual responses for edges collinear to the motion path during specific phases of brisk eye-movements, namely saccadic exploration or large changes of gaze between distant fixation locations. This unexpected process could account for the observation of transient peaks of responses for fast-moving contours coaligned with the RF axis (Barry Richmond, personal communication; Judge et al. 1980) and the induction of filling-in responses for fast centripetal radial flow (Troncoso et al. 2015).

\section{Conclusion}

We conclude from this review that the functional complexity in the early visual system is largely underestimated and that the functional organization and preference expressed in visual cortical RFs result from the coordination by input statistics dynamics of overlaid activity processes operating at different spatial integration scales. We have illustrated here what insight can be possibly gained by the comparison between different levels of integration. Reverse engineering on intracellular and spiking signals shows that part of the "effective" connectivity contributing to the RF is missed/ignored when models and data collection are confined at the spiking level. Mapping of the hidden non-linearities in the subthreshold RF reveals unexpected immergence processes, driven by the stimulus, through which the global activity control extending within and beyond the cortical hypercolumn regulates the functional expression of more microscopic properties, such as the apparent "Simpleness" of individual RFs. This feature can be seen as a top-down influence of the more mesoscopic levels of organization, typical of complex dynamic systems based on nested processing. The unfortunate consequence of this physiological finding for modellers is that one can no longer hope or pretend to simulate the full network behavior by assembling neurons with fixed intrinsic or context-invariant properties in a pure bottom-up approach. Models of the early visual system have to incorporate homeostasis rules acting across integration levels to account for the inverse covariation between input drive complexity and the 
apparent linearity of the more elementary processing units. In a kind of reciprocal way, it remains nevertheless highly plausible that the characterization of psychic laws at a more holistic level, guiding the psychological principles of low-level (non attentive) perception, pleads for the existence of structuro-functional traces that can be retrieved by decoding and averaging synaptic echoes. The identification at more microscopic scales of the key non-linearities and the activity synergy requirements necessary for their expression should help us to understand, in a causal way, the emergence of novel binding principles at more mesoscopic levels, leading eventually to the abstract formalization of psychological foundations of perception.

Acknowledgments Work was supported by CNRS, The Paris-Saclay IDEX (NeuroSaclay and I-Code), the French National Research Agency (ANR: NatStats and Complex-V1) and the European Community (FET-Bio-I3 integrated programs (IP FP6: FACETS (015879), IP FP7: BRAINSCALES (269921); FET-Open (Brain-i-nets (243914); FET-Flagship: The Human Brain Project).

Open Access This chapter is distributed under the terms of the Creative Commons AttributionNoncommercial 2.5 License (http://creativecommons.org/licenses/by-nc/2.5/) which permits any noncommercial use, distribution, and reproduction in any medium, provided the original author(s) and source are credited.

The images or other third party material in this chapter are included in the work's Creative Commons license, unless indicated otherwise in the credit line; if such material is not included in the work's Creative Commons license and the respective action is not permitted by statutory regulation, users will need to obtain permission from the license holder to duplicate, adapt or reproduce the material.

\section{References}

Alonso JM (2002) Neural connections and receptive field properties in the primary visual cortex. Neuroscientist 8(5):443-456

Bai EW, Cerone V, Regruto D (2007) Separable inputs for the identification of block-oriented non-linear systems. In: Proceedings of 2007 American Control Conference, New York, pp $1548-1553$

Barlow HB (1972) Single units and sensation: a neuron doctrine for perceptual psychology? Perception 1:371-394

Baudot P, Levy M, Marre O, Monier C, Pananceau M, Frégnac Y (2013) Animation of natural scene by virtual eye-movements evokes high precision and low noise in V1 neurons. Front Neural Circ 7:206-235

Benucci A, Frazor RA, Carandini M (2007) Standing waves and traveling waves distinguish two circuits in visual cortex. Neuron 55(1):103-117

Borg-Graham LJ, Monier C, Frégnac Y (1998) Visual input evokes transient and strong shunting inhibition in visual cortical neurons. Nature 393:369-373

Bosking WH, Zhang Y, Schofield B, Fitzpatrick D (1997) Orientation selectivity and the arrangement of horizontal connections in tree shrew striate cortex. J Neurosci 1(6):2112-2127

Bringuier V, Chavane F, Glaeser L, Frégnac Y (1999) Horizontal propagation of visual activity in the synaptic integration field of area 17 neurons. Science 283:695-699

Buzàs P, Kovács K, Ferecskó AS, Budd JM, Eysel UT, Kisvárday ZF (2006) Model-based analysis of excitatory lateral connections in the visual cortex. J Comp Neurol 499(6):861-881

Callaway EM, Katz LC (1990) Emergence and refinement of clustered horizontal connections in cat striate cortex. J Neurosci 10(4):1134-1153 
Carandini M, Demb JB, Mante V, Tolhurst DJ, Dan Y, Olshausen BA (2005) Do we know what the early visual system does? J Neurosci 25(46):10577-10597

Chance FS, Nelson SB, Abbott LF (1999) Complex cells as cortically amplified simple cells. Nat Neurosci 2:277-282

Chavane F, Sharon D, Jancke D, Marre O, Frégnac Y, Grinvald A (2011) Lateral spread of orientation selectivity in V1 is controlled by intracortical cooperativity. Front Syst Neurosci 5:4-24. doi:10.3389/fnsys.2011.00004

Chen X, Han F, Poo M-M, Dan Y (2007) Excitatory and suppressive receptive field subunits in awake monkey primary visual cortex (V1). Proc Natl Acad Sci USA 104:19120-19125

Douglas RJ, Martin KA (2004) Neuronal circuits of the neocortex. Annu Rev Neurosci 27:419-451

Ferster D, Miller KD (2000) Neural mechanisms of orientation selectivity in the visual cortex. Annu Rev Neurosci 23:441-471

Field DJ, Hayes A, Hess RF (1993) Contour integration by the human visual system: evidence for a local "association field". Vision Res 33(2):173-193

Fournier J, Monier C, Pananceau M, Frégnac Y (2011) Adaptation of the simple or complex nature of V1 receptive fields to visual statistics. Nat Neurosci 14(8):1053-1060

Fournier Y, Monier C, Levy M, Marre O, Sari K, Kisvarday ZF, Frégnac Y (2014) Hidden complexity of synaptic receptive fields in cat primary visual cortex. J Neurosci 34(16):5515-5528

Frégnac Y (2012) Reading out the synaptic echoes of low level perception in V1. Lect Notes Comput Sci 7583:486-495

Frégnac Y, Rudolph M, Davison A, Destexhe A (2007) Complexity and level hierarchy in neural networks. In: Képès $F$ (ed) Biological networks. Complex systems and interdisciplinary science series. World Scientific, Singapore, pp 291-340

Frégnac Y, Bathellier B (2015) Cortical correlates of low-level perception: from neural circuits to percepts. Neuron 88:110-126

Gérard-Mercier F, Carelli P, Pananceau M, Baudot P, Troncoso X, Frégnac Y (2014) A saccadic view of the "silent surround" of visual cortical receptive fields. American Society for Neuroscience Abstracts, Washington, DC

Gerard-Mercier F, Pananceau M, Carelli P, Troncoso X, Frégnac Y (2016) Synaptic correlates of low-level perception in V1. J Neurosci (submitted)

Gilbert CD, Li W (2012) Adult visual cortical plasticity. Neuron 75(2):250-264

Gilbert CD, Wiesel TN (1983) Clustered intrinsic connections in cat visual cortex. J Neurosci 3 (5):1116-1133

Haider B, Krause MR, Duque A, Yu Y, Touryan J, Mazer JA, McCormick DA (2010) Synaptic and network mechanisms of sparse and reliable visual cortical activity during nonclassical receptive field stimulation. Neuron 65(1):107-121

Henry GH (1977) Receptive field classes of cells in the striate cortex of the cat. Brain Res 133:1-28

Huang X, Elyada YM, Bosking WH, Walker T, Fitzpatrick D (2014) Optogenetic assessment of horizontal interactions in primary visual cortex. J Neurosci 2(34):4976-4990 [Erratum in J Neurosci 34(26): 8930]

Hubel DH, Wiesel TN (1962) Receptive fields, binocular interaction and functional architecture in the cat's visual cortex. J Physiol (London) 160:106-154

Hubel DH, Wiesel TN (1968) Receptive fields and functional architecture of monkey striate cortex. J Physiol (London) 195:215-243

Hubel DH, Wiesel TN (2005) Brain and visual perception. Oxford University Press, New York

Jones HE, Grieve KL, Wang W, Sillitio AM (1980) Surround suppression in primate V1. J Neurophysiol 86:2011-2028

Judge SJ, Wurtz RH, Richmond BJ (1980) Vision during saccadic eye movements. I. Visual interactions in striate cortex. J Neurophysiol 43(4):1133-1155

Kasamatsu T, Miller R, Zhu Z, Chang M, Ishida Y (2010) Collinear facilitation is independent of receptive field expansion at low contrast. Exp Brain Res 201(3):453-465

Kisvardày ZF, Toth E, Rausch M, Eysel UT (1997) Orientation-specific relationship between populations of excitatory and inhibitory lateral connections in the visual cortex of the cat. Cereb Cortex 7:605-618

Li W, Piëch V, Gilbert CD (2006) Contour saliency in primary visual cortex. Neuron 50(6):951-962 
Li W, Piëch V, Gilbert CD (2008) Learning to link visual contours. Neuron 57(3):442-451

Martin KAC (2014) Obituary David Hubel (1926-2013). Curr Biol 24(1):R4-R7

Martin KAC, Roth S, Rusch ES (2014) Superficial layer pyramidal cells communicate heterogeneously between multiple functional domains of cat primary visual cortex. Nat Commun 5:5252-5265. doi:10.1038/ncomms6252

Mizobe K, Polat U, Pettet MW (2001) Facilitation and suppression of single striate-cell activity by spatially discrete pattern stimuli presented beyond the receptive field. Vis Neurosci 18 (3):377-391

Monier C, Chavane F, Baudot P, Graham LJ, Frégnac Y (2003) Orientation and direction selectivity of synaptic activity in visual cortical neurons: a diversity of combinations produces spike tuning. Neuron 37:663-680

Monier C, Fournier J, Frégnac Y (2008) In vitro and in vivo measures of evoked excitatory and inhibitory conductance dynamics in sensory cortices. J Neurosci Methods 169:323-365

Nauhaus I, Busse L, Carandini M, Ringach DL (2009) Stimulus contrast modulates functional connectivity in visual cortex. Nat Neurosci 12:70-76

Olshausen BA (2014) 20 years of learning about vision: questions answered, questions unanswered, and questions not yet asked. In: Bower JM (ed) 20 years of computational neuroscience. Springer, New York. ISBN 978-1-4614-1424-7

Polat U, Sagi D (1985) Lateral interactions between spatial channels: suppression and facilitation revealed by lateral masking experiments. Vision Res 33(7):993-999

Polat U, Sagi D (1993) Lateral interactions between spatial channels: suppression and facilitation revealed by lateral masking experiments. Vision Res 33:993-999

Priebe NJ, Ferster D (2012) Mechanisms of neuronal computation in mammalian visual cortex. Neuron 75(2): 194-208

Priebe NJ, Mechler F, Carandini M, Ferster D (2004) The contribution of spike threshold to the dichotomy of cortical simple and complex cells. Nat Neurosci 7(10):1113-1122

Rust NC, Schwartz O, Movshon JA, Simoncelli EP (2005) Spatiotemporal elements of macaque v1 receptive fields. Neuron 46:945-956

Sillito AM, Jones HE (1996) Context-dependent interactions and visual processing in V1. J Physiol (Paris) 90(3-4):205-209

Sillito AM, Grieve KL, Jones HE, Davis J (1995) Visual cortical mechanisms detecting focal orientation discontinuities. Nature 378(6556):492-496

Skottun BC, De Valois RL, Grosof DH, Movshon JA, Albrecht DG, Bonds AB (1991) Classifying simple and complex cells on the basis of response modulation. Vision Res 31:1079-1086

Tan AYY, Chen Y, Scholl B, Seidemann E, Priebe NJ (2014) Sensory stimulation shifts cortex from synchronous to asynchronous states. Nature 509(8):226-230

Tao L, Shelley M, McLaughlin D, Shapley R (2004) An egalitarian network model for the emergence of simple and complex cells in visual cortex. Proc Natl Acad Sci USA 101:366-371

Touryan J, Lau B, Dan Y (2002) Isolation of relevant visual features from random stimuli for cortical complex cells. J Neurosci 22(24):10811-10818

Touryan J, Felsen G, Dan Y (2005) Spatial structure of complex cell receptive fields measured with natural images. Neuron 45:781-791

Troncoso X, Pananceau M, Lebec B, Desbois C, Gerard-Mercier F, Frégnac Y (2015) Spatial synergy and temporal coherence requirements for binding the feedforward and horizontal waves in V1. American Society for Neuroscience Abstracts, Chicago, IL

Vinje WE, Gallant JL (2000) Sparse coding and decorrelation in primary visual cortex during natural vision. Science 287(5456):1273-1276

Wagemans J, Edler JH, Kubovy M, Palmer SE, Peterson MA, Singh M, von der Heydt R (2012) A century of Gestalt psychology in visual perception. I. Perceptual grouping and figure-ground organization. Psychol Bull 183(6):1172-1217

Yeh CI, Xing D, Williams PE, Shapley RM (2009) Stimulus ensemble and cortical layer determine V1 spatial receptive fields. Proc Natl Acad Sci USA 106:14652-14657 